\title{
Low Intensity of Running Favors for Anabolic Response after Resistance Exercise
}

Chun-Chung Chou ${ }^{1}$; Siang-Sheng $\mathrm{Wu}^{2}$; Te-Sheng Chen ${ }^{3}$; Jung-Charng Lin ${ }^{1}$

${ }^{1}$ Chinese Culture University; ${ }^{2}$ National Taiwan Normal University; ${ }^{3}$ National

Taiwan Sport University

Classification of First Author (Doctoral Student)

\section{Abstract}

Purpose: Whether active or passive recovery after resistance exercise may affect anabolic and catabolic response is not clear. The aim of this study was to examine the effect of active (moderate or low intensity running) and passive (rest) recovery after resistance exercise on testosterone, cortisol and testosterone/cortisol ratio ( $\mathrm{T} / \mathrm{C})$ responses. Methods: By counter-balanced design, nine recreationally active males (age: $23.89 \pm 0.86$ yrs of age; height: $172.89 \pm 1.30 \mathrm{~cm}$; weight: $68.37 \pm 2.72 \mathrm{~kg} ; \dot{\mathrm{V}} \mathrm{O}_{2} \max : 56.56 \pm 1.70 \mathrm{ml} / \mathrm{kg} / \mathrm{min}$ ) completed three tests including: $65 \% \dot{\mathrm{V}}_{2}$ max running (moderate intensity, $\mathrm{RM}$ ), $40 \% \mathrm{~V}_{2}$ max running (low intensity, $\mathrm{RL}$ ) and passive rest (RR) following 3sets of four resistance exercises (bench press, leg extension, front lat pulldown and leg curl, 12 repetitions at $60 \% 1 R M$ with 2 min rest among all sets and exercises). In order to evaluate the systemic anabolic status, we measured plasma testosterone, cortisol and $\mathrm{T} / \mathrm{C}$ ratio before resistance exercise (baseline), immediately after either active or passive recovery (post), and 30min after recovery (rest). Results: In RM group, T/C was significantly lower (decrease $18.5 \%, p<.05$ ) than baseline at rest. In $\mathrm{RL}$ group, $\mathrm{T} / \mathrm{C}$ was significantly higher (increase $79.1 \%$ at post and increase $123.2 \%$ at rest, $p<.05$ ) than baseline at post and rest, respectively. At rest, the $T / C$ of $R L$ was higher than RM (RM: $0.49 \pm 0.12, \mathrm{RL}: 1.08 \pm 0.19, \mathrm{p}<.05$ ). Conclusions: Resistance exercise and low intensity aerobic exercise is more favorable to enhance anabolic response during post-exercise recovery.

Keywords: testosterone, cortisol, T/C ratio, aerobic exercise 